TRANSACTIONS OF THE

AMERICAN MATHEMATICAL SOCIETY

Volume 361, Number 8, August 2009, Pages 4125-4142

S 0002-9947(09)04693-5

Article electronically published on February 23, 2009

\title{
DEGENERATION OF A-INFINITY MODULES
}

\author{
BERNT TORE JENSEN, DAG MADSEN, AND XIUPING SU
}

\begin{abstract}
In this paper we use $A_{\infty}$-modules to study the derived category of a finite dimensional algebra over an algebraically closed field. We study varieties parameterising $A_{\infty}$-modules. These varieties carry an action of an algebraic group such that orbits correspond to quasi-isomorphism classes of complexes in the derived category. We describe orbit closures in these varieties, generalising a result of Zwara and Riedtmann for modules.
\end{abstract}

\section{INTRODUCTION}

In this paper we study parameter spaces of objects in the derived category of a finite dimensional algebra $\Lambda$ over an algebraically closed field $k$.

The derived category of $\Lambda$ can be defined by formally inverting all quasi-isomorphisms in the category of complexes of $\Lambda$-modules. Recall that a quasi-isomorphism is a chain map which induces isomorphism in homology. So two isomorphic objects in the derived category have isomorphic homologies, but the converse is in general not true. A natural point of view is therefore that quasi-isomorphism classes are determined by the isomorphism type of their homology and some extra information which explains how the homology is put together to form a complex. In this paper we will take this point of view.

We study families of objects in the derived category with fixed dimensions or even fixed isomorphism type in homology. We do so by replacing the ordinary derived category with the equivalent derived category of $A_{\infty}$-modules $[\mathrm{K}]$. The equivalence functor preserves homology, so dimensions and isomorphism types are not affected. The $A_{\infty}$-modules are called polydules in [L-H], and we use both names in this paper.

Keller has observed $\left[\mathrm{K}\right.$ that by fixing dimensions in homology $\underline{d}=\left(d_{a}, \ldots, d_{b}\right)$ we obtain a variety, here denoted by $\mathcal{M}=\mathcal{M}_{\Lambda, \underline{d}}$, which parameterises polydules with homology dimensions $\underline{d}$. There is an algebraic group $\mathcal{G}=\mathcal{G}_{\underline{d}}$ acting on $\mathcal{M}_{\Lambda, \underline{d}}$ such that orbits correspond to quasi-isomorphism classes of polydules. It is this variety and group action we examine in the present paper.

The main result is a generalisation of a result of Zwara $\underline{\mathrm{Z}}$ and Riedtmann $[\underline{\mathrm{R}}$ for module varieties to these varieties of polydules.

Received by the editors May 29, 2007.

2000 Mathematics Subject Classification. Primary 18E30; Secondary 14L30, 16 G10.

Key words and phrases. A-infinity modules, degeneration (orbit closure), derived categories.

This research was supported by the European Research Training Network LIEGRITS (MRTNCT-2003-505078). This work was partly done during a visit at the Université de Sherbrooke. All three authors wish to thank the representation theory group in Sherbrooke for their hospitality. The third author would like to thank the Swiss Science Foundation for financial support during her stay at the University of Berne 2005/2006.

(c)2009 American Mathematical Society Reverts to public domain 28 years from publication 
Theorem. Let $M, N \in \mathcal{M}_{\Lambda, \underline{d}}$. Then $N \in \overline{\mathcal{G} \cdot M}$ if and only if there is a finite dimensional $\Lambda$-polydule $Z$ and an exact triangle

$$
Z \rightarrow M \oplus Z \rightarrow N \rightarrow Z[1] .
$$

In [JSZ, the first and third authors of the present paper together with Zimmermann developed another theory of degeneration in derived categories. There degenerations are studied in the space comproj ${ }^{d}$, a space which parameterises right bounded complexes of projective modules with so-called dimension array $\underline{\mathrm{d}}$. Both approaches, the one in [JSZ and in the one in the present paper, have their advantages and may serve different purposes. We make a more detailed comparison between the two approaches in section 5. Here in the Introduction we only emphasise the fact that $\mathcal{M}_{\Lambda, \underline{d}}$ is an affine variety, while comproj- in general is only an inverse limit of affine varieties.

The paper is organised as follows. In section 1 we recall the definition of $A_{\infty^{-}}$ modules and their morphisms. In section 2 we give a summary of the most important known results concerning $A_{\infty}$-modules and their derived category. In section 3 we describe in detail the variety $\mathcal{M}$ and the group $\mathcal{G}$ with its action on $\mathcal{M}$. We consider the special cases where the homology is given as representations of a quiver with relations or where the homology module structure is fixed in section 4 . In section 5 we prove our main result, an algebraic characterisation of degeneration.

\section{A-INFinity MOdules}

In this section we give the basic definitions of $A_{\infty}$-modules and their morphisms. We stress that our definitions are adapted to the case we are interested in, namely $A_{\infty}$-modules over associative algebras, and are only valid for this case. For the definitions in the most general case $\left(A_{\infty}\right.$-modules over $A_{\infty}$-algebras), we refer to $[\mathrm{K}]$ and $[\mathrm{L}-\mathrm{H}$.

For our purposes we find it most practical to use a left module notation. A (left) $A_{\infty}$-module over an algebra $\Lambda$ in our sense is the same as a (right) $A_{\infty}$-module over $\Lambda^{\mathrm{op}}$ in the sense of $[\mathrm{K}]$. Since all our algebra elements are in degree 0 we avoid certain sign issues.

1.1. Modules. Let $k$ be a commutative ring and $\Lambda$ an associative $k$-algebra. An $A_{\infty}$-module over $\Lambda$ (or a $\Lambda$-polydule $[\mathrm{L}$ - $\mathrm{H}]$ ) is a graded $k$-module

$$
M=\bigoplus_{i \in \mathbb{Z}} M^{i}
$$

with $k$-linear graded maps

$$
m_{n}: \Lambda^{\left(\otimes_{k}\right) n-1} \otimes_{k} M \rightarrow M, n \geq 1,
$$

of degree $n-2$ satisfying the rules

$$
\begin{aligned}
& m_{1} m_{1}=0, \\
& m_{1} m_{2}=m_{2}\left(1 \otimes m_{1}\right),
\end{aligned}
$$

and for $n \geq 3$

$$
\sum_{i=1}^{n}(-1)^{i(n-1)} m_{n-i+1}\left(1^{\otimes n-i} \otimes m_{i}\right)=\sum_{j=1}^{n-2}(-1)^{j-1} m_{n-1}\left(1^{\otimes n-j-2} \otimes \mu \otimes 1^{\otimes j}\right),
$$

where 1 is the identity map and $\mu$ is the multiplication in $\Lambda$. 
In particular $\left(M, m_{1}\right)$ is a complex of $k$-modules. Note that if $M$ is bounded, then $m_{n}=0$ for $n \gg 0$.

1.2. Change of base rings. Suppose $M=\bigoplus_{i \in \mathbb{Z}} M^{i}$ is a $\Lambda$-polydule with maps $m_{n}^{M}, n \geq 1$. If $V$ is an (ungraded) $k$-module, then the graded $k$-module

$$
M \otimes_{k} V=\bigoplus_{i \in \mathbb{Z}}\left(M^{i} \otimes_{k} V\right)
$$

is a $\Lambda$-polydule with maps

$$
m_{n}^{M \otimes V}=m_{n}^{M} \otimes 1_{V}: \Lambda^{\left(\otimes_{k}\right) n-1} \otimes_{k} M \otimes_{k} V \rightarrow M \otimes_{k} V, n \geq 1 .
$$

Suppose now that $\theta: k \rightarrow k^{\prime}$ is a morphism of commutative rings. Then $\Lambda \otimes_{k} k^{\prime}$ is a $k^{\prime}$-algebra, and a $\Lambda \otimes_{k} k^{\prime}$-polydule is a graded $k^{\prime}$-module $N=\bigoplus_{i \in \mathbb{Z}} N^{i}$ with $k^{\prime}$-linear maps

$$
m_{n}^{N}:\left(\Lambda \otimes_{k} k^{\prime}\right)^{\left(\otimes_{k^{\prime}}\right) n-1} \otimes_{k^{\prime}} N \rightarrow N, n \geq 1,
$$

satisfying the equations. Since

$$
\left(\Lambda \otimes_{k} k^{\prime}\right)^{\left(\otimes_{k^{\prime}}\right) n-1} \otimes_{k^{\prime}} N \simeq \Lambda^{\left(\otimes_{k}\right) n-1} \otimes_{k} N,
$$

this structure is determined by $k^{\prime}$-linear maps

$$
m_{n}^{N}: \Lambda^{\left(\otimes_{k}\right) n-1} \otimes_{k} N \rightarrow N, n \geq 1 .
$$

The map $\theta: k \rightarrow k^{\prime}$ induces a $\Lambda$-polydule structure on $N$.

If $M=\bigoplus_{i \in \mathbb{Z}} M^{i}$ is a $\Lambda$-polydule, there is a $\Lambda \otimes_{k} k^{\prime}$-polydule structure on the graded $k^{\prime}$-module

$$
M \otimes_{k} k^{\prime}=\bigoplus_{i \in \mathbb{Z}}\left(M^{i} \otimes_{k} k^{\prime}\right)
$$

given by the $k^{\prime}$-linear maps

$$
m_{n}^{M \otimes k^{\prime}}=m_{n}^{M} \otimes 1: \Lambda^{\left(\otimes_{k}\right) n-1} \otimes_{k} M \otimes_{k} k^{\prime} \rightarrow M \otimes_{k} k^{\prime}, n \geq 1 .
$$

1.3. Morphisms and compositions. A morphism $f: L \rightarrow N$ between two $\Lambda$ polydules $L$ and $N$ is given by a family of maps

$$
f_{n}: \Lambda^{\left(\otimes_{k}\right) n-1} \otimes_{k} L \rightarrow N, n \geq 1,
$$

of degree $n-1$ satisfying the rules

$$
\begin{aligned}
f_{1} m_{1} & =m_{1} f_{1}, \\
f_{1} m_{2}-f_{2}\left(1 \otimes m_{1}\right) & =m_{2}\left(1 \otimes f_{1}\right)+m_{1} f_{2},
\end{aligned}
$$

and for $n \geq 3$

$$
\begin{aligned}
\sum_{i=1}^{n}(-1)^{i(n-1)} f_{n-i+1}\left(1^{\otimes n-i} \otimes m_{i}\right) & +\sum_{j=1}^{n-2}(-1)^{j} f_{n-1}\left(1^{\otimes n-j-2} \otimes \mu \otimes 1^{\otimes j}\right) \\
& =\sum_{r=1}^{n}(-1)^{(r+1) n} m_{n-r+1}\left(1^{\otimes n-r} \otimes f_{r}\right) .
\end{aligned}
$$

Note in particular that $f_{1}$ is a chain map $f_{1}:\left(L, m_{1}\right) \rightarrow\left(N, m_{1}\right)$ between complexes of $k$-modules. A morphism is called a quasi-isomorphism if $f_{1}:\left(L, m_{1}\right) \rightarrow\left(N, m_{1}\right)$ is a quasi-isomorphism of complexes. 
A morphism $f$ is called strict if $f_{i}=0$ for $i>1$. Furthermore $f$ is called a strict monomorphism if $f$ is strict and $f_{1}$ is a monomorphism. Similarly $f$ is called a strict epimorphism if $f$ is strict and $f_{1}$ is an epimorphism.

The identity morphism $1_{N}: N \rightarrow N$ is given by $f_{1}=1$ and $f_{i}=0$ for all $i>0$. The composition $f g: N \rightarrow M$ of two morphisms $f: L \rightarrow M$ and $g: N \rightarrow L$ is given by the rule

$$
(f g)_{n}=\sum_{i=1}^{n}(-1)^{(i-1) n} f_{n-i+1}\left(1^{\otimes n-i} \otimes g_{i}\right) .
$$

A morphism $f: L \rightarrow N$ is called null-homotopic if there is a family of maps

$$
h_{n}: \Lambda^{\left(\otimes_{k}\right) n-1} \otimes_{k} L \rightarrow N, n \geq 1,
$$

of degree $-n$ such that

$$
\begin{aligned}
& f_{1}=m_{1} h_{1}+h_{1} m_{1}, \\
& f_{2}=m_{1} h_{2}-m_{2}\left(1 \otimes h_{1}\right)-h_{2}\left(1 \otimes m_{1}\right)+h_{1} m_{2},
\end{aligned}
$$

and for $n \geq 3$

$$
\begin{aligned}
f_{n}= & \sum_{r=1}^{n}(-1)^{r(n-1)} m_{n-r+1}\left(1^{\otimes n-r} \otimes h_{r}\right) \\
& +\sum_{i=1}^{n}(-1)^{i(n-1)} h_{n-i+1}\left(1^{\otimes n-i} \otimes m_{i}\right) \\
& +\sum_{j=1}^{n-2}(-1)^{j} h_{n-1}\left(1^{\otimes n-j-2} \otimes \mu \otimes 1^{\otimes j}\right) .
\end{aligned}
$$

Two morphisms $f$ and $g$ from $L$ to $N$ are called homotopic if $f-g$ is nullhomotopic. In this case we say that $h$ is a homotopy between $f$ and $g$. A morphism $f: L \rightarrow N$ is called a homotopy equivalence if there exists a morphism $g: N \rightarrow L$ such that $g f$ is homotopic to $1_{L}$ and $f g$ is homotopic to $1_{N}$.

1.4. Unitality. A $\Lambda$-polydule $M$ is called strictly unital if for all $a \in M, m_{2}(1, a)=$ $a$ and $m_{n}\left(\lambda_{1}, \ldots, \lambda_{n-1}, a\right)=0$ if $n \geq 3$ and $1 \in\left\{\lambda_{1}, \ldots, \lambda_{n-1}\right\}$.

A morphism $f: L \rightarrow N$ is called strictly unital if $f_{n}\left(\lambda_{1}, \ldots, \lambda_{n-1}, a\right)=0$ whenever $n \geq 2$ and $1 \in\left\{\lambda_{1}, \ldots, \lambda_{n-1}\right\}$. Similarly a homotopy $h$ between two morphisms $f$ and $g$ is called strictly unital if $h_{n}\left(\lambda_{1}, \ldots, \lambda_{n-1}, a\right)=0$ whenever $n \geq 2$ and $1 \in\left\{\lambda_{1}, \ldots, \lambda_{n-1}\right\}$. If there is a strictly unital homotopy between $f$ and $g$, we denote this fact by $f \sim_{h} g$.

\section{THE DERIVED CATEGORY}

Suppose now that $k$ is a field and $\Lambda$ is a $k$-algebra. In this section we give a summary of the, for our purposes, most important results from $\mathrm{K}$ and $\mathrm{L}-\mathrm{H}$ concerning $A_{\infty}$-modules and their derived category.

Let $\operatorname{Mod}_{\infty} \Lambda$ denote the category of strictly unital $\Lambda$-polydules and strictly unital morphisms. The homotopy category $\mathcal{H}_{\infty}(\Lambda)$ is defined to be the quotient category $\mathcal{H}_{\infty}(\Lambda)=\operatorname{Mod}_{\infty} \Lambda / \sim_{h}$, so morphisms in $\mathcal{H}_{\infty}(\Lambda)$ are homotopy classes of morphisms in $\operatorname{Mod}_{\infty} \Lambda$. The following theorem is of central importance.

Theorem 2.1 ([ $\mathrm{K}, 4.2]$, $[\mathrm{L}-\mathrm{H}, 2.4 .1 .1])$. A morphism $f: L \rightarrow N$ is a homotopy equivalence if and only if it is a quasi-isomorphism. 
This gives us one of several essentially equivalent ways of defining the derived category $\mathcal{D}_{\infty}(\Lambda)$ (see section 2.4.2 in $[\mathrm{L}-\mathrm{H}]$ ). We simply define it to be the same as the homotopy category, so $\mathcal{D}_{\infty}(\Lambda)=\mathcal{H}_{\infty}(\Lambda)$.

The category $\mathcal{D}_{\infty}(\Lambda)$ is a triangulated category. The shift of a $\Lambda$-polydule $M$, denoted $M[1]$, is the $\Lambda$-polydule defined by $(M[1])^{i}=M^{i+1}$ and $m_{n}^{M[1]}=(-1)^{n} m_{n}^{M}$. If $f: L \rightarrow N$ is a morphism in $\mathcal{D}_{\infty}(\Lambda)$, then there exists an object denoted cone $f$ and called the cone of $f$, and an exact triangle

$$
L \stackrel{f}{\rightarrow} N \stackrel{j}{\rightarrow} \text { cone } f \stackrel{p}{\rightarrow} L[1]
$$

in $\mathcal{D}_{\infty}(\Lambda)$. The cone has graded parts

$$
(\text { cone } f)^{i}=N^{i} \oplus(L[1])^{i},
$$

and the $\Lambda$-polydule structure is defined by

$$
m_{n}^{\text {cone } f}=\left(\begin{array}{cc}
m_{n}^{N} & (-1)^{n} f_{n} \\
0 & m_{n}^{M[1]}
\end{array}\right) .
$$

Every exact triangle in $\mathcal{D}_{\infty}(\Lambda)$ is isomorphic to one whose form is as above. The morphism $j$ is a strict monomorphism, the morphism $p$ is a strict epimorphism and the sequence of graded $k$-vector spaces $0 \rightarrow N \stackrel{j_{1}}{\longrightarrow}$ cone $f \stackrel{p_{1}}{\longrightarrow} L[1] \rightarrow 0$ is exact.

Using the rotation axiom, we get the same description of exact triangles as in [K, 5.2]. Let $L \stackrel{j}{\rightarrow} M \stackrel{p}{\rightarrow} N$ be a sequence with $j$ a strict monomorphism, with $p$ a strict epimorphism and where the sequence of graded $k$-vector spaces $0 \rightarrow L \stackrel{j_{1}}{\longrightarrow} M \stackrel{p_{1}}{\longrightarrow} N \rightarrow 0$ is exact. We call such a sequence an exact sequence of $\Lambda$-polydules. In this case there is an exact triangle $L \stackrel{j}{\rightarrow} M \stackrel{p}{\rightarrow} N \rightarrow L[1]$ in $\mathcal{D}_{\infty}(\Lambda)$, so cone $j \simeq N$ in $\mathcal{D}_{\infty}(\Lambda)$. All exact triangles are obtained in this way.

If $(C, \delta)$ is a complex of $\Lambda$-modules, then $C$ can be viewed as a $\Lambda$-polydule where $m_{1}=\delta$, the map $m_{2}: \Lambda \otimes C \rightarrow C$ is given by the $\Lambda$-module structure and $m_{n}=0$ for $n \geq 3$. Identifying complexes with polydules in this way induces a triangle functor $\mathcal{D}(\operatorname{Mod} \Lambda) \rightarrow \mathcal{D}_{\infty}(\Lambda)$, where $\mathcal{D}(\operatorname{Mod} \Lambda)$ is the usual (unbounded) derived category of $\Lambda$. It turns out that this functor is an equivalence.

Theorem $2.2([\mathrm{~K}, 4.3],[\mathrm{L}-\mathrm{H}, 2.4 .3 .2])$. The canonical functor $\mathcal{D}(\operatorname{Mod} \Lambda) \rightarrow \mathcal{D}_{\infty}(\Lambda)$ is an equivalence of triangulated categories.

In other words each quasi-isomorphism class of $\Lambda$-polydules corresponds to (has as a subclass) exactly one quasi-isomorphism class of complexes of $\Lambda$-modules. So every polydule is quasi-isomorphic to a complex in this sense.

In this paper, however, we will focus on another type of polydule, the one with $m_{1}=0$. A good thing about such objects is that quasi-isomorphisms between them are invertible already in $\operatorname{Mod}_{\infty} \Lambda$.

Theorem $2.3([\mathbb{K}, 4.3])$. Suppose $M$ and $N$ are two $\Lambda$-polydules with $m_{1}^{M}=0=$ $m_{1}^{N}$. Then $M$ and $N$ are quasi-isomorphic if and only they are isomorphic.

Each quasi-isomorphism class of polydules has an object with $m_{1}=0$, so polydules of this type can be chosen as representatives of isomorphism classes in the derived category $\mathcal{D}_{\infty}(\Lambda)$.

Theorem 2.4 ([L-H, 3.3.1.7]). Suppose $M$ is a strictly unital $\Lambda$-polydule. Then there is a strictly unital $\Lambda$-polydule $M^{\prime}$ with $m_{1}^{M^{\prime}}=0$ and a strictly unital quasiisomorphism $M^{\prime} \rightarrow M$. 
If $M$ is a complex, meaning $m_{n}^{M}=0$ for $n \geq 3$, then the graded parts of $M^{\prime}$ are the homology modules of $\left(M, m_{1}^{M}\right)$. In general if $N$ is a $\Lambda$-polydule with $m_{1}^{N}=0$, then each $N^{i}, i \in \mathbb{Z}$, is a $\Lambda$-module in its own right where the module structure is $m_{2}^{N}$ restricted to $m_{2}^{N}: \Lambda \oplus N^{i} \rightarrow N^{i}$. It is also worth noting that if $f: M \rightarrow N$ is a morphism and $m_{1}^{M}=0=m_{1}^{N}$, then for each $i \in \mathbb{Z}$, the map $f_{1}: M^{i} \rightarrow N^{i}$ is a $\Lambda$-module morphism. A polydule with $m_{1}=0$ can informally be thought of as "homology + structure".

Our aim is to study the bounded derived category of finitely generated $\Lambda$ modules, $\mathcal{D}^{b}(\bmod \Lambda)$, when $\Lambda$ is a finite dimensional $k$-algebra. The category $\mathcal{D}^{b}(\bmod \Lambda)$ is in this case equivalent to the full subcategory of $\mathcal{D}(\operatorname{Mod} \Lambda)$ consisting of objects with finite dimensional total homology. We get the following characterisation of the corresponding polydules.

Proposition 2.5. Let $\Lambda$ be a finite dimensional k-algebra. Then a $\Lambda$-polydule $M$ is in the essential image of the composed functor $\mathcal{D}^{b}(\bmod \Lambda) \hookrightarrow \mathcal{D}(\operatorname{Mod} \Lambda) \stackrel{\sim}{\longrightarrow}$ $\mathcal{D}_{\infty}(\Lambda)$ if and only if it is quasi-isomorphic to a $\Lambda$-polydule $M^{\prime}$ with $m_{1}^{M^{\prime}}=0$ and $\operatorname{dim}_{k} M^{\prime}<\infty$.

We denote by $\mathcal{D}_{\infty}^{f}(\Lambda)$ the full subcategory of $\mathcal{D}_{\infty}(\Lambda)$ consisting of objects in this image. Obviously we have an equivalence of triangulated categories $\mathcal{D}^{b}(\bmod \Lambda) \stackrel{\sim}{\longrightarrow}$ $\mathcal{D}_{\infty}^{f}(\Lambda)$.

\section{DESCRIPTION OF VARIETY}

In this section we describe the geometric object we want to study. Suppose that $k$ is an algebraically closed field and $\Lambda$ is a finite dimensional $k$-algebra. We keep these assumptions for the rest of the paper. We fix dimensions in homology, nonzero only on a finite interval, and make a variety out of the possible $\Lambda$-polydule structures with $m_{1}=0$ and the given homology dimensions. Via the equivalence $\mathcal{D}^{b}(\bmod \Lambda) \stackrel{\sim}{\longrightarrow} \mathcal{D}_{\infty}^{f}(\Lambda)$, we can view this as a parameter space of objects in the derived category with the given homology dimensions. There is a group of quasiisomorphisms acting on this space. As noted by Keller [ $[\mathrm{K}, 4.3]$, what we have in this situation is an algebraic group acting on an algebraic variety. While this section is devoted to definitions, in section 5 we pursue our main goal, namely to find an algebraic characterisation of orbit closure (degeneration).

Suppose $\mathcal{B}=\left\{\nu_{1}=1, \nu_{2}, \ldots, \nu_{t}\right\}$ is a $k$-basis of $\Lambda$. We fix integers $a<b$ and a finite dimensional graded $k$-vector space $V=\bigoplus_{i=a}^{b} V^{i}$. We want to construct a variety $\mathcal{M}$ of possible $A_{\infty}$-module structures on $V$. Let $d_{i}=\operatorname{dim}_{k} V^{i}$ for $a \leq i \leq b$, and let $\underline{d}$ denote the vector $\underline{d}=\left(d_{a}, \ldots, d_{b}\right)$. We fix bases for the graded parts of $V$, so that maps between them are represented by matrices.

A point in the variety $\mathcal{M}=\mathcal{M}_{\Lambda, \underline{d}}$ is a collection of matrices $M(S, i)$, one for each sequence $S=\left(\lambda_{|S|}, \ldots, \lambda_{2}, \lambda_{1}\right)$ of $1 \leq|S| \leq b-a+1$ elements from $\mathcal{B}$ and $i$ a number satisfying $a+|S|-1 \leq i \leq b$. The matrix $M(S, i)$ is of size $d_{i-|S|+1} \times d_{i}$ and represents the map

$$
m_{|S|+1}\left(\lambda_{|S|}, \ldots, \lambda_{1},-\right): V^{i} \rightarrow V^{i-|S|+1} .
$$

We consider only strictly unital polydules, so if $\nu_{1}=1 \in S$, then

$$
M(S, i)= \begin{cases}I & \text { if }|S|=1, \\ 0 & \text { if }|S|>1 .\end{cases}
$$


Given a sequence $S=\left(\lambda_{|S|}, \ldots, \lambda_{1}\right)$ and $1 \leq l \leq|S|-1$, there are uniquely determined elements $c_{r}^{S, l} \in k, 1 \leq r \leq t$, satisfying the expression

$$
\lambda_{l+1} \lambda_{l}=\sum_{r=1}^{t} c_{r}^{S, l} \nu_{r}
$$

The map

$$
m_{|S|}\left(\lambda_{|S|}, \ldots, \lambda_{l+2}, \lambda_{l+1} \lambda_{l}, \lambda_{l-1}, \ldots, \lambda_{1},-\right): V^{i} \rightarrow V^{i-|S|+2}
$$

is represented by the matrix $\sum_{r=1}^{t} c_{r}^{S, l} M\left(S_{l / r}, i\right)$, where $S_{l / r}$ is the sequence

$$
S_{l / r}=\left(\lambda_{|S|}, \ldots, \lambda_{l+2}, \nu_{r}, \lambda_{l-1}, \ldots, \lambda_{1}\right)
$$

of length $|S|-1$ obtained from $S$ by deleting $\lambda_{l+1}$ and $\lambda_{l}$ and inserting $\nu_{r}$ in position $l$ from the right.

The matrices $M(S, i)$ have to satisfy the relations

$\sum_{S=\left[S^{\prime}, S^{\prime \prime}\right]}(-1)^{|S|\left(\left|S^{\prime \prime}\right|+1\right)} M\left(S^{\prime}, i-\left|S^{\prime \prime}\right|+1\right) \cdot M\left(S^{\prime \prime}, i\right)=\sum_{l=1}^{|S|-1} \sum_{r=1}^{t}(-1)^{l-1} c_{r}^{S, l} \cdot M\left(S_{l / r}, i\right)$

(this and other formulas in this section are obtained from the corresponding formulas in section 1), where the sum on the left hand side is taken over all decompositions of $S$ into two parts $S^{\prime}=\left(\lambda_{|S|}, \ldots, \lambda_{\left|S^{\prime \prime}\right|+1}\right)$ and $S^{\prime \prime}=\left(\lambda_{\left|S^{\prime \prime}\right|}, \ldots, \lambda_{1}\right)$ with $\left|S^{\prime}\right|,\left|S^{\prime \prime}\right| \geq 1$. We have one such relation for each pair $(S, i)$ with $2 \leq|S| \leq b-a+2$ and $a+|S|-2 \leq i \leq b$.

Remark 3.1. If $a=b=0$ and $\underline{d}=(d)$, then as a special case $\mathcal{M}_{\Lambda, \underline{d}}$ is equal to the ordinary module variety $\bmod (\Lambda, d)$. Using the action by generators, such module varieties can easily be defined whenever $\Lambda$ is a (not necessarily finite dimensional) finitely generated algebra. For $\Lambda$-polydules however, the structure maps $m_{n+1}\left(\zeta_{n}, \ldots, \zeta_{1},-\right): M \rightarrow M$ with the $\zeta_{i}, 1 \leq i \leq n$, taken from a given set of generators of $\Lambda$ do not always determine the whole $\Lambda$-polydule structure on $M$. Our construction of the variety $\mathcal{M}_{\Lambda, \underline{d}}$ depends on the fact that $\Lambda$ has a finite $k$-basis. It is not known to us which infinite dimensional algebras $\Lambda$ have well-defined varieties of $\Lambda$-polydules, but for such varieties to exist, the algebra needs to satisfy a stronger condition than finite generation.

Next we describe the group $\mathcal{G}=\mathcal{G}_{\underline{d}}$ of quasi-isomorphisms. An element $g$ in $\mathcal{G}$ is a collection of matrices $F(S, i)$, one for each sequence $S$ of $0 \leq|S| \leq b-a$ elements and $i$ a number satisfying $a+|S| \leq i \leq b$. Note that here we allow $S$ to be the empty sequence $\varnothing$. The matrix $F(S, i)$ is of size $d_{i-|S|} \times d_{i}$ and is thought of as representing a map

$$
f_{|S|+1}\left(\lambda_{|S|}, \ldots, \lambda_{1},-\right): V^{i} \rightarrow V^{i-|S|} .
$$

We only want to consider strictly unital morphisms, so we add the condition that $F(S, i)=0$ whenever $1 \in S$. Since $f_{1}: V \rightarrow V$ is supposed to be a quasiisomorphism, the matrices $F(\varnothing, i)$ must be invertible for all $a \leq i \leq b$. These are the only conditions for $g$ to be an element in $\mathcal{G}$.

The product $g=(F(S, i))$ of two group elements $g^{\prime}=\left(F^{\prime}(S, i)\right)$ and $g^{\prime \prime}=$ $\left(F^{\prime \prime}(S, i)\right)$ is given by the rule

$$
F(S, i)=\sum_{S=\left[S^{\prime}, S^{\prime \prime}\right]}(-1)^{\left|S^{\prime}\right|\left|S^{\prime \prime}\right|} F^{\prime}\left(S^{\prime}, i-|S|\right) \cdot F^{\prime \prime}\left(S^{\prime \prime}, i\right) .
$$


The identity element $e$ of this group is the element given by $F(\varnothing, i)=I_{d_{i}}$ for all $i$ and $F(S, i)=0$ for all $S \neq \varnothing$. The inverse $g^{-1}=\left(F^{\prime}(S, i)\right)$ of an element $g=(F(S, i))$ is given recursively by

$$
F^{\prime}(\varnothing, i)=F(\varnothing, i)^{-1}
$$

and

$$
F^{\prime}(S, i)=\left[\sum_{S=\left[S^{\prime}, S^{\prime \prime}\right], S^{\prime \prime} \neq \varnothing}(-1)^{\left|S^{\prime}\right|\left|S^{\prime \prime}\right|+1} F^{\prime}\left(S^{\prime}, i-|S|\right) \cdot F\left(S^{\prime \prime}, i\right)\right] F(\varnothing, i)^{-1}
$$

for all $S \neq \varnothing$.

We now describe the action of $\mathcal{G}$ on $\mathcal{M}$. The map $*: \mathcal{G} \times \mathcal{M} \rightarrow \mathcal{M}$ is given in such a way that if $g=(F(S, i))$ and $x=(M(S, i))$, then $x^{\prime}=g * x$ is given by

$$
\begin{aligned}
M^{\prime}(S, i)= & \sum_{S=\left[S^{\prime}, S^{\prime \prime}\right], S^{\prime \prime} \neq \varnothing}(-1)^{\left|S^{\prime}\right|\left(\left|S^{\prime \prime}\right|+1\right)} F\left(S^{\prime}, i-\left|S^{\prime \prime}\right|+1\right) \cdot M\left(S^{\prime \prime}, i\right) \\
& +\sum_{l=1}^{|S|-1} \sum_{r=1}^{t}(-1)^{l-1} c_{r}^{S, l} \cdot F\left(S_{l / r}, i\right) \\
& \left.+\sum_{S=\left[S^{\prime}, S^{\prime \prime}\right], S^{\prime}, S^{\prime \prime} \neq \varnothing}(-1)^{\left|S^{\prime}\right|\left|S^{\prime \prime}\right|+1} M^{\prime}\left(S^{\prime}, i-|S|\right) \cdot F\left(S^{\prime \prime}, i\right)\right] F(\varnothing, i)^{-1}
\end{aligned}
$$

The $\mathcal{G}$-orbits in $\mathcal{M}$ correspond to isomorphism classes of objects in $\mathcal{D}^{b}(\bmod \Lambda)$ with the given dimensions in homology. We define degeneration in $\mathcal{M}$ in the usual way as follows. Let $M$ and $N$ be two $\Lambda$-polydules with $m_{1}^{M}=0=m_{1}^{N}$ and the given homology dimensions. We use the same letters to denote the corresponding points in $\mathcal{M}$. If $N$ belongs to the Zariski closure of the $\mathcal{G}$-orbit of $M$, that is, $N \in \overline{\mathcal{G} \cdot M}$, we say that $M$ degenerates to $N$ and we denote this fact by $M \leq_{\operatorname{deg}} N$. The relation $\leq_{\mathrm{deg}}$ is reflexive and transitive by elementary properties of the closure operation. Since $\mathcal{G} \cdot M$ is a constructible set, it contains a subset $U$ which is open and dense in $\overline{\mathcal{G} \cdot M}$. Therefore if $N \in \overline{\mathcal{G} \cdot M}$ and $M \in \overline{\mathcal{G} \cdot N}$, then $\mathcal{G} \cdot M=\mathcal{G} \cdot N$ and $M \simeq N$. The conclusion is that the relation $\leq_{\mathrm{deg}}$ is a partial order on the set of isomorphism classes of $\Lambda$-polydules with $m_{1}=0$ and the given homology dimensions.

\section{VARIATIONS AND SIMPLIFICATIONS}

The variety $\mathcal{M}$ with its action by $\mathcal{G}$ has a rather complicated definition which is not so easy to work with in general. In this section we consider some special cases of algebras and polydules where we can define smaller and simpler spaces and group actions.

Let $\bmod (\Lambda, \underline{d}) \subseteq \mathcal{M}_{\Lambda, \underline{d}}$ be the subvariety consisting of polydules with $M(S, i)=0$ for all $S$ with $|S|>1$. That is, each polydule in $\bmod (\Lambda, \underline{d})$ is a graded $\Lambda$-module, and $\bmod (\Lambda, \underline{d})$ is isomorphic to a product of module varieties

$$
\bmod (\Lambda, \underline{d}) \cong \prod_{i} \bmod \left(\Lambda, d_{i}\right)
$$

There is a projection map

$$
\pi: \mathcal{M}_{\Lambda, \underline{d}} \rightarrow \bmod (\Lambda, \underline{d})
$$


defined by $\pi(M(S, i))=0$ for all $S$ with $|S|>1$. Here $M$ and $N$ belong to the same fibre if and only if they have equal homology as graded modules. That is, $\pi(M)=H^{*} M$ computes the homology of a polydule.

The group $\mathrm{GL}_{\underline{d}}=\prod_{i} \mathrm{GL}_{d_{i}}$ acts on $\bmod (\Lambda, \underline{d})$ by conjugation, and the orbits are in bijection with isomorphism classes of graded $\Lambda$-modules with graded dimension $\underline{d}$. There is a morphism

$$
\mathcal{G} \rightarrow \mathrm{GL}_{\underline{d}}
$$

of algebraic groups mapping all $F(S, i)$ with $|S|>0$ to zero. We let $\mathcal{G}$ act on $\bmod (\Lambda, d)$ via this morphism. Then by the definition of the action of $\mathcal{G}$ on $\mathcal{M}$, the projection $\pi$ is $\mathcal{G}$-equivariant.

4.1. Quivers with relations. A very important combinatorial tool for studying modules over finite dimensional algebras is to use quivers with relations. We shall see that quivers are also useful when studying polydules.

Now assume that $\Lambda=k Q / I$ for a quiver $Q=\left(Q_{0}, Q_{1}\right)$ and an admissible ideal $I \subseteq k Q$. Such algebras are called quiver algebras. It is well known that the category of $\Lambda$-modules is equivalent to the category of representations of $Q$ satisfying the relations in $I$ (see ARS for an introduction to quivers and their representations). The change of point of view from modules to quiver representations can be regarded as a change of the underlying monoidal base category from $k$-modules to $\left(k^{\times r}\right)$ modules, where $r=\left|Q_{0}\right|$. In the same way we can obtain a quiver representation version of polydules, as we now explain.

Throughout $[\mathrm{L}-\mathrm{H}]$, as explicitly stated in the beginning of its first chapter, the theory developed is valid for more general monoidal base categories than $k$-modules. We give the details of the more general framework (taken from section 2.1 of $[\mathrm{L}-\mathrm{H}]$ ) and explain how quiver representations fit into this. Let $\left(\mathrm{C}, \otimes_{\mathrm{C}}, e_{\mathrm{C}}\right)$ be a semisimple monoidal Grothendieck $k$-category and let $C^{\prime}$ be a semi-simple Grothendieck $k$-category. Let $\mathrm{Gr}^{\prime}$ denote the category of graded $\mathrm{C}^{\prime}$-objects

$$
M=\left(M^{i}\right)_{i \in \mathbb{Z}}, M^{i} \text { in } \mathrm{C}^{\prime} .
$$

Suppose there is an action of $C$ on $C^{\prime}$ given by a bifunctor $\diamond: C \times C^{\prime} \rightarrow C^{\prime}$ with isomorphisms $\Psi=\Psi_{X Y M}:\left(X \otimes_{\mathrm{C}} Y\right) \diamond M \stackrel{\sim}{\longrightarrow} X \diamond(Y \diamond M)$ and $\Upsilon=\Upsilon_{M}: e_{\mathrm{C}} \diamond M \stackrel{\sim}{\longrightarrow} M$ natural in $X, Y$ in $C$ and $M$ in $C^{\prime}$. These natural isomorphisms are supposed to satisfy the usual coherence conditions $\mathrm{JK}$. The action of $\mathrm{C}$ on $\mathrm{C}^{\prime}$ extends to an action on $\mathrm{Gr} \mathrm{C}^{\prime}$.

Let $\Lambda$ be an algebra in $\mathrm{C}$, that is, an object $\Lambda$ in $\mathrm{C}$ together with an associative multiplication map $\mu: \Lambda \otimes_{\mathrm{C}} \Lambda \rightarrow \Lambda$ and a unit map $\iota: e_{\mathrm{C}} \rightarrow \Lambda$ satisfying $\mu\left(\iota \otimes_{\mathrm{C}} \Lambda\right)=$ $1_{\Lambda}=\mu(\Lambda \otimes \mathrm{C} \iota)$. A $\Lambda$-polydule in $\mathrm{C}^{\prime}$ is a graded $\mathrm{C}^{\prime}$-object $M$ with $k$-linear graded maps

$$
m_{n}: \Lambda^{(\otimes c) n-1} \diamond M \rightarrow M, n \geq 1,
$$

of degree $n-2$ satisfying the same rules as the maps $m_{n}$ in section 1 The corresponding modifications are also made to the definitions of morphisms and homotopies.

The general framework applies to quiver representations as follows. Let $\mathrm{C}$ be the semi-simple monoidal Grothendieck $k$-category of $\left(k^{\times r}\right)-\left(k^{\times r}\right)$-bimodules and let $\mathrm{C}^{\prime}$ be the semi-simple Grothendieck $k$-category of left $\left(k^{\times r}\right)$-modules. The action $\diamond=\bigotimes_{(k \times r)}: \mathrm{C} \times \mathrm{C}^{\prime} \rightarrow \mathrm{C}^{\prime}$ satisfies the conditions above. A quiver algebra $\Lambda=k Q / I$ can be viewed as an algebra in $C$. A $\Lambda$-polydule in $C^{\prime}$ is the same as a graded representation of $Q$ with an $A_{\infty}$-structure respecting the quiver. We call such a 
graded representation a polyrepresentation. Every part of the theory of polydules developed in $[\mathrm{L}-\mathrm{H}]$ is in particular applicable to polyrepresentations. For instance we have the following analogue of Theorem 2.2 .

Theorem 4.1 ([L-H, 2.4.3.2]). The derived category of polyrepresentations is triangle equivalent to the derived category of representations.

Obviously the derived category of representations is again triangle equivalent to the derived category of $\Lambda$-modules. We also need the following analogue of Theorem 2.3 .

Theorem 4.2 ([L-H, 3.3.1.7]). Suppose $M$ is a strictly unital $\Lambda$-polyrepresentation. Then there is a strictly unital $\Lambda$-polyrepresentation $M^{\prime}$ with $m_{1}^{M^{\prime}}=0$ and a strictly unital quasi-isomorphism $M^{\prime} \rightarrow M$.

We now define a variety of polyrepresentations. We fix not only dimensions in homology, but also dimension vectors. So the given data is a sequence $\underline{e}=$ $\left(\underline{e}^{a}, \ldots, \underline{e}^{b}\right)$ of dimension vectors $\underline{e}^{i}=\left(e_{1}^{i}, \ldots, e_{r}^{i}\right), a \leq i \leq b$. Let $\underline{d}=\left(d_{a}, \ldots, d_{b}\right)$, where $d_{i}=\sum_{j \in Q_{0}} e_{j}^{i}$. Assume a basis for $\Lambda$ is chosen that consists only of paths. We define a subvariety

$$
\operatorname{Rep}_{\infty}(\Lambda, \underline{e}) \subseteq \mathcal{M}_{\Lambda, \underline{d}}
$$

as follows. For each point $x=(M(S, i)) \in \mathcal{M}_{\Lambda, \underline{d}}$, divide each matrix $M(S, i)$ into blocks $C_{j, l}(S, i), 1 \leq j, l \leq r$, of size $e_{l}^{i-|S|+1} \times e_{j}^{i}$. The subvariety $\operatorname{Rep}_{\infty}(\Lambda, \underline{e})$ consists of all $x \in \mathcal{M}_{\Lambda, \underline{d}}$ which satisfy all of the following three conditions:

(i) $M(S, i)=0$ if the composition $\lambda_{|S|} \cdots \lambda_{1}$ of the paths in $S$ is not a path in $Q$.

(ii) $C_{j, l}(S, i)=0$ if the composition $\lambda_{|S|} \cdots \lambda_{1}$ of the paths in $S$ is a path in $Q$ starting in vertex $s$ and ending in vertex $t$ and $(j, l) \neq(s, t)$.

(iii) $M(S, i)=0$ if $|S|>1$ and $S$ contains a path of length zero.

The variety $\operatorname{Rep}_{\infty}(\Lambda, \underline{e})$ is the variety of polyrepresentations whose sequence of dimension vectors is $\underline{e}=\left(\underline{e}^{a}, \ldots, \underline{e}^{b}\right)$.

The image of $\operatorname{Rep}_{\infty}(\Lambda, \underline{e})$ under $\pi$,

$$
\pi\left(\operatorname{Rep}_{\infty}(\Lambda, \underline{e})\right)=\operatorname{Rep}(\Lambda, \underline{e}) \subseteq \bmod (\Lambda, \underline{d}),
$$

is the variety of graded representations of $Q$ satisfying the relations in $I$ whose sequence of dimension vectors is $\underline{e}=\left(\underline{e}^{a}, \ldots, \underline{e}^{b}\right)$. For each $a \leq i \leq b$, a further projection to the $i$ th component gives

$$
\operatorname{Rep}\left(\Lambda, \underline{e}^{i}\right) \subseteq \bmod \left(\Lambda, d_{i}\right)
$$

the variety of representations of $Q$ satisfying the relations in $I$ with dimension vector $\underline{e}^{i}$.

For each element $g=(F(S, i)) \in \mathcal{G}_{\underline{d}}$, divide each matrix $F(S, i)$ into blocks $D_{j, l}(S, i), 1 \leq j, l \leq r$, of size $e_{l}^{i-|S|} \times e_{j}^{i}$. Let $\mathcal{G}_{Q}=\mathcal{G}_{Q, \underline{e}}$ be the subset of $\mathcal{G}_{\underline{d}}$ consisting of the elements $g \in \mathcal{G}_{\underline{d}}$ which satisfy all of the following four conditions:

(i) $D_{j, l}(\varnothing, i)=0$ if $j \neq l$.

(ii) $F(S, i)=0$ if $|S|>0$ and the composition $\lambda_{|S|} \cdots \lambda_{1}$ of the paths in $S$ is not a path in $Q$.

(iii) $D_{j, l}(S, i)=0$ if $|S|>0$, the composition $\lambda_{|S|} \cdots \lambda_{1}$ of the paths in $S$ is a path in $Q$ starting in vertex $s$ and ending in vertex $t$ and $(j, l) \neq(s, t)$.

(iv) $F(S, i)=0$ if $|S|>0$ and $S$ contains a path of length zero. 
The set $\mathcal{G}_{Q}$ is the set of quasi-isomorphisms which respect the quiver, which are exactly the quasi-isomorphisms in the category of polyrepresentations. It can easily be seen that $\mathcal{G}_{Q} \subseteq \mathcal{G}$ is a subgroup. So if two polyrepresentations $M$ and $N$ belong to the same quasi-isomorphism class (of polyrepresentations), then there are quasiisomorphisms belonging to $\mathcal{G}_{Q}$ between them in both directions. It follows that $\mathcal{G}_{Q^{-}}$ orbits in $\operatorname{Rep}_{\infty}(\Lambda, \underline{e})$ correspond to quasi-isomorphism classes of polyrepresentations and therefore to isomorphism classes of objects in $\mathcal{D}^{b}(\bmod \Lambda)$.

For each $M \in \mathcal{M}_{\Lambda, \underline{d}}$, there is a unique sequence of dimension vectors $\underline{e}=$ $\left(\underline{e}^{a}, \ldots, \underline{e}^{b}\right)$ such that $M \in \mathcal{G} \cdot \operatorname{Rep}_{\infty}(\Lambda, \underline{e})$. So $\mathcal{M}_{\Lambda, \underline{d}}$ is the disjoint union of the sets $\mathcal{G} \cdot \operatorname{Rep}_{\infty}(\Lambda, \underline{e})$, where we let $\underline{e}$ vary over the finitely many possible sequences of dimension vectors. Each set $\mathcal{G} \cdot \operatorname{Rep}_{\infty}(\Lambda, \underline{e})$ is closed and therefore also open. Moreover, each set $\mathcal{G} \cdot \operatorname{Rep}_{\infty}(\Lambda, \underline{e})$ is connected. This follows, since each polydule degenerates to its homology (as we prove below) and each module degenerates to the direct sum of its composition factors. The conclusion is that the connected components of $M \in \mathcal{M}_{\Lambda, \underline{d}}$ are exactly the sets of the form $\mathcal{G} \cdot \operatorname{Rep}_{\infty}(\Lambda, \underline{e})$.

We give a short argument showing that a polydule degenerates to its homology.

Lemma 4.3. Let $M \in \mathcal{M}_{\Lambda, \underline{d}}$. Then $M \leq_{\operatorname{deg}} H^{*}(M)$.

Proof. Let $M_{t}$ be the polydule given by $M_{t}(S, i)=t^{|S|-1} M(S, i)$ for all $|S|>1$. We have a strict isomorphism $M_{t} \cong M$ for all $t \neq 0$ and $M_{0}=H^{*}(M)$. Therefore $M \leq_{\operatorname{deg}} H^{*}(M)$.

The following lemma states that the degeneration of polyrepresentations is equivalent to the degeneration of polydules. We postpone the proof until the end of section 5 .

Lemma 4.4. Let $M, N \in \operatorname{Rep}_{\infty}(\Lambda, \underline{e})$. Then $M \leq_{\operatorname{deg}} N$ if and only if $N \in \overline{\mathcal{G}_{Q} \cdot M}$.

4.2. Polydules with fixed homology. Let $X \in \bmod (\Lambda, \underline{d})$ be a graded module with graded dimension $\underline{d}$. Let $\mathcal{M}_{X}=\pi^{-1}(X)$ be the variety of polydules with fixed homology $X$. Let $\mathcal{G}_{X}$ be the setwise stabilizer of $\mathcal{M}_{X}$, consisting of group elements with $F(\varnothing, i) \in \operatorname{Aut}_{\Lambda}\left(H^{i}(X)\right)$. There is a bijection between the orbits under the induced action of $\mathcal{G}_{X}$ on $\mathcal{M}_{X}$ and the isomorphism classes of polydules with homology equal to $X$. The variety $\mathcal{M}_{X}$ is connected. Its unique closed orbit is the orbit of $X$. The following lemma may be proven in a similar way as Lemma 4.4 above. We skip the details.

Lemma 4.5. Let $M, N \in \mathcal{M}_{X}$. Then $M \leq_{\operatorname{deg}} N$ if and only if $N \in \overline{\mathcal{G}_{X} \cdot M}$.

As a special case we can consider polydules with fixed homology in two degrees. That is, $X=M[a] \oplus N[b]$ for two $\Lambda$-modules $M$ and $N$ and degrees $a$ and $b$. Then the equations defining $\mathcal{M}_{X}$ are affine equations, and therefore $\mathcal{M}_{X}$ is an affine space. The following lemma is a useful application of varieties of polydules to the problem of checking the vanishing of extension spaces between $\Lambda$-modules.

Lemma 4.6. Let $M$ and $N$ be two finitely generated $\Lambda$-modules and let $X=$ $M \oplus N[i]$, where $i \geq 1$. Then $\operatorname{Ext}_{\Lambda}^{i+1}(M, N)=0$ if and only if $\mathcal{G}_{X} \cdot X=\mathcal{M}_{X}$.

Proof. We have $\operatorname{Ext}_{\Lambda}^{i+1}(M, N)=\operatorname{Hom}(M, N[i+1]) \simeq \operatorname{Hom}(M[-1], N[i])$. If $f$ : $M[-1] \rightarrow N[i]$ is a morphism, then the cone of $f$ is in $\mathcal{M}_{X}$ and every point in $\mathcal{M}_{X}$ can be obtained as the cone of such a morphism. The cone of $f$ is quasi-isomorphic to $X$ if and only if $f$ is null-homotopic. The lemma follows. 
Suppose $\Lambda=k Q / I$ for a quiver $Q=\left(Q_{0}, Q_{1}\right)$ and an admissible ideal $I \subseteq k Q$. We can combine the constructions in this section and consider polyrepresentations with fixed homology. Let $X \in \operatorname{Rep}(\Lambda, \underline{e})$ be a graded representation with sequence of dimension vectors $\underline{e}=\left(\underline{e}^{a}, \ldots, \underline{e}^{b}\right)$. Then define

$$
\mathcal{M}_{X}^{Q}=\mathcal{M}_{X} \cap \operatorname{Rep}_{\infty}(\Lambda, \underline{e})
$$

or equivalently $\mathcal{M}_{X}^{Q}=\left(\pi^{\prime}\right)^{-1}(X)$, where $\pi^{\prime}$ is the restricted function

$$
\pi^{\prime}=\left.\pi\right|_{\operatorname{Rep}_{\infty}(\Lambda, \underline{e})}: \operatorname{Rep}_{\infty}(\Lambda, \underline{e}) \rightarrow \operatorname{Rep}(\Lambda, \underline{e}) .
$$

There is a group $\mathcal{G}_{X}^{Q}=\mathcal{G}_{X} \cap \mathcal{G}_{Q, \underline{e}}$ acting on $\mathcal{M}_{X}^{Q}$ and orbits correspond to isomorphism classes of polyrepresentations. Analogues of Lemmas 4.5 and 4.6 hold in this situation.

Lemma 4.7. Let $M, N \in \mathcal{M}_{X}^{Q}$. Then $M \leq_{\operatorname{deg}} N$ if and only if $N \in \overline{\mathcal{G}_{X}^{Q} \cdot M}$.

Lemma 4.8. Suppose $\Lambda=k Q / I$ for a quiver $Q=\left(Q_{0}, Q_{1}\right)$ and an admissible ideal $I \subseteq k Q$. Let $M$ and $N$ be two finitely generated $\Lambda$-modules and let $X=M \oplus N[i]$, where $i \geq 1$. Then $\operatorname{Ext}_{\Lambda}^{i+1}(M, N)=0$ if and only if $\mathcal{G}_{X}^{Q} \cdot X=\mathcal{M}_{X}^{Q}$.

\section{Characterisation of orbit closure}

In this section we prove our main result, an algebraic characterisation of degeneration (orbit closure) in the variety $\mathcal{M}_{\Lambda, \underline{d}}$. Keeping in mind the equivalence $\mathcal{D}^{b}(\bmod \Lambda) \stackrel{\sim}{\longrightarrow} \mathcal{D}_{\infty}^{f}(\Lambda)$, this should be seen as a derived category version of a celebrated theorem by Zwara [Z] and Riedtmann [R]. In fact the Riedtmann-Zwara Theorem is a special case of the result we prove here.

In [GO'H] the authors gave a very general theorem characterising orbit closures in varieties. It takes the following form when applied to our situation.

Theorem 5.1. Let $M, N \in \mathcal{M}_{\Lambda, \underline{d}}$. Then $M \leq_{\operatorname{deg}} N$ if and only if there is a discrete valuation $k$-algebra $R$ (residue field $k$, and quotient field $K$ finitely generated and of transcendence degree one over $k$ ) and a $\Lambda \otimes_{k} R$-polydule structure with $m_{1}=0$ on the graded $R$-module $Q=\bigoplus_{i=a}^{b} R^{d_{i}}$ such that $Q \otimes_{R} k=N$ (via the identification $R \otimes_{R} k=k$ ) and $Q \otimes_{R} K \simeq M \otimes_{k} K$ as $\Lambda \otimes_{k} K$-polydules.

Proof. The statement follows from [GO'H, 1.2].

Using this as a starting point, we can prove the "Zwara direction" of our characterisation. Zwara $[\mathrm{Z}]$ was the first to prove the analogous statement for module varieties. Yoshino $[\mathrm{Y}]$ defined module degeneration for any $k$-algebra even when there is no underlying variety, and showed that Zwara's result was still valid in this generality [Y, 2.2]. Our proof, although completely different due to the triangulated setting, borrows some ideas from $[\mathrm{Y}$.

Theorem 5.2 ("Zwara direction"). Let $M, N \in \mathcal{M}_{\Lambda, \underline{d}}$. If $M \leq_{\operatorname{deg}} N$, then there is an object $Z$ in $\mathcal{D}_{\infty}^{f}(\Lambda)$ and an exact triangle

$$
Z \rightarrow M \oplus Z \rightarrow N \rightarrow Z[1] .
$$

Proof. Suppose $M \leq_{\operatorname{deg}} N$. Let $R$ be a discrete valuation $k$-algebra as in Theorem 5.1 and let $Q=\bigoplus_{i=a}^{b} R^{d_{i}}$ be a $\Lambda \otimes_{k} R$-polydule such that $m_{1}^{Q}=0, Q \otimes_{R} k=N$ and $Q \otimes_{R} K \simeq M \otimes_{k} K$ as $\Lambda \otimes_{k} K$-polydules. Let $t$ denote a generator of the maximal ideal in $R$. 
Let $i: M \otimes_{k} R \rightarrow M \otimes_{k} K$ be the strict inclusion of $\Lambda \otimes_{k} R$-polydules and let $f$ be the composition

$$
M \otimes_{k} R \stackrel{i}{\rightarrow} M \otimes_{k} K \stackrel{\sim}{\rightarrow} Q \otimes_{R} K .
$$

Since $M \otimes_{k} R$ and $Q \otimes_{R} K$ have support in only a finite number of degrees, we know that $f_{i}=0$ for $i>>0$. Since in addition $M \otimes_{k} R$ is finitely generated as an $R$ module, there is an $n$ such that the morphism $f$ factors through the strict inclusion $Q \otimes_{R}\left(1 / t^{n}\right) R \rightarrow Q \otimes_{R} K$. There is a strict isomorphism $Q \otimes_{R}\left(1 / t^{n}\right) R \simeq Q$. We have the following morphism of triangles in $\mathcal{D}_{\infty}(\Lambda)$ :

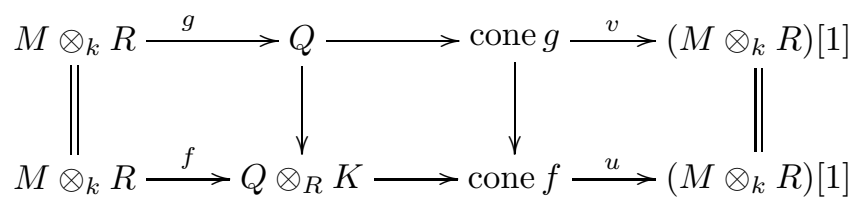

Since $f$ is a section, we have $u=0$ by [ㅍ, I.4.1]. Since $u=0$, then also $v=0$ and $g$ is a section. It follows that $g_{1}: M \otimes_{k} R \rightarrow Q$ is injective. Since $M \otimes_{k} R$ and $Q$ have the same rank over $R$, it follows by the construction of the cone that cone $g$ has finite dimensional total homology.

Now consider the strict inclusion of $\Lambda$-polydules $j: M \otimes_{k} t R \rightarrow M \otimes_{k} R$. From the exact sequence of vector spaces $0 \rightarrow t R \rightarrow R \rightarrow k \rightarrow 0$, we get an exact sequence of $\Lambda$-polydules $0 \rightarrow M \otimes_{k} t R \stackrel{j}{\rightarrow} M \otimes_{k} R \rightarrow M \rightarrow 0$, so $M \simeq$ cone $j$ in $\mathcal{D}_{\infty}(\Lambda)$. By the octahedral axiom, we get the following diagram of exact (marked by $\triangle$ ) and commuting triangles in $\mathcal{D}_{\infty}(\Lambda)$ :
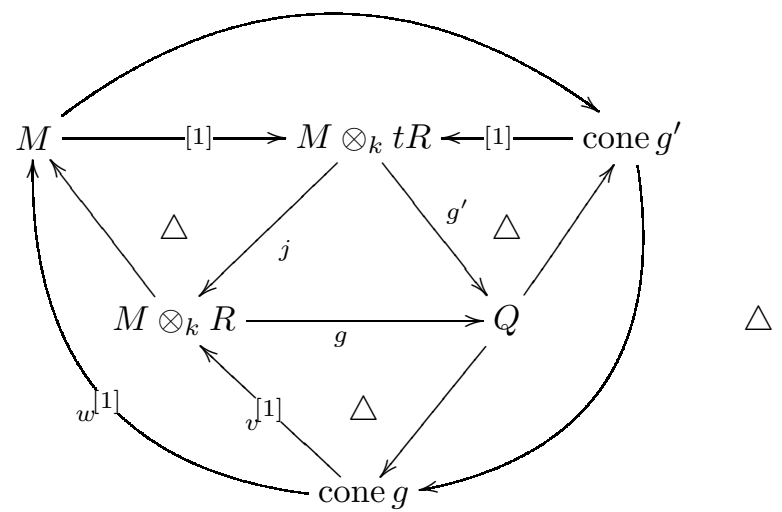

So there is an exact triangle $M \rightarrow$ cone $g^{\prime} \rightarrow$ cone $g \stackrel{w}{\longrightarrow} M[1]$. Since $v=0$, then also $w=0$ and cone $g^{\prime} \simeq M \oplus$ cone $g$.

From the commutative square

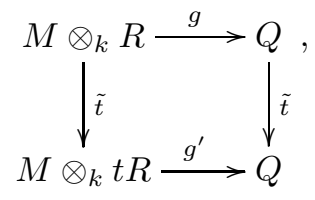


where $\tilde{t}$ denotes multiplication by $t$, we get the commutative diagram

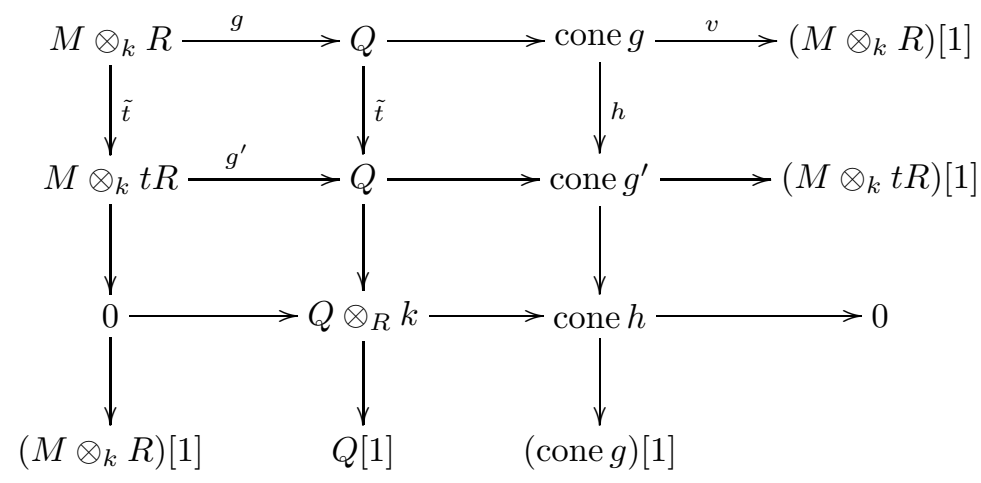

where the rows and columns are exact triangles $\mathrm{W}$, Ex.10.2.6.]. We see that cone $h \simeq Q \otimes_{R} k=N$ and that there is an exact triangle

$$
\text { cone } g \stackrel{h}{\rightarrow} \text { cone } g^{\prime} \rightarrow \text { cone } h \rightarrow(\text { cone } g)[1]
$$

in $\mathcal{D}_{\infty}(\Lambda)$. We put $Z=$ cone $g$, which is an object in $\mathcal{D}_{\infty}^{f}(\Lambda)$. The triangle above is isomorphic to $Z \rightarrow M \oplus Z \rightarrow N \rightarrow Z[1]$.

In order to prove the converse of this theorem, we first make the useful observation that the existence of an exact triangle of the form in question implies the existence of a certain exact sequence of polydules.

Lemma 5.3. Let $M, N \in \mathcal{M}_{\Lambda, d}$. Suppose there is an exact triangle of the form $Z \rightarrow M \oplus Z \rightarrow N \rightarrow Z[1]$ in $\mathcal{D}_{\infty}^{f}(\Lambda)$.

Then there is an exact sequence of finite dimensional $\Lambda$-polydules of the form $0 \rightarrow Z \stackrel{f}{\rightarrow} M^{\prime} \oplus Z^{\prime} \stackrel{g}{\rightarrow} N \rightarrow 0$ with $m_{1}^{Z}=m_{1}^{Z^{\prime}}=m_{1}^{M^{\prime}}=0$ and where $Z \simeq Z^{\prime}$ and $M \simeq M^{\prime}$. (These isomorphisms are possibly non-strict.)

Proof. Suppose there is a triangle $Z \rightarrow M \oplus Z \rightarrow N \stackrel{h}{\rightarrow} Z[1]$ in $\mathcal{D}_{\infty}^{f}(\Lambda)$. We may assume that $Z$ is represented in such a way that $m_{1}^{Z}=0$. The morphism $h[-1]: N[-1] \rightarrow Z$ gives rise to an exact sequence $0 \rightarrow Z \stackrel{f}{\rightarrow} \operatorname{cone}(h[-1]) \stackrel{g}{\rightarrow} N \rightarrow 0$. Since $\operatorname{dim}_{k} M^{i}=\operatorname{dim}_{k} N^{i}$ for all $i \in \mathbb{Z}$, then also

$$
\operatorname{dim}_{k}(M \oplus Z)^{i}=\operatorname{dim}_{k}(\operatorname{cone}(h[-1]))^{i}
$$

for all $i \in \mathbb{Z}$. The quasi-isomorphism $M \oplus Z \rightarrow \operatorname{cone}(h[-1])$ must therefore be a (possibly non-strict) isomorphism and $m_{1}^{\operatorname{cone}(h[-1])}=0$.

We are now ready to prove the converse of Theorem 5.2, Our proof is similar to Riedtmann's original proof for module varieties [ $\underline{\mathrm{R}}, 3.4]$.

Theorem 5.4 ("Riedtmann direction"). Let $M, N \in \mathcal{M}_{\Lambda, \underline{d}}$. If there is an exact triangle $Z \rightarrow M \oplus Z \rightarrow N \rightarrow Z[1]$ in $\mathcal{D}_{\infty}^{f}(\Lambda)$, then $M \leq_{\operatorname{deg}} N$.

Proof. Suppose there is a triangle $Z \rightarrow M \oplus Z \rightarrow N \stackrel{h}{\rightarrow} Z[1]$ in $\mathcal{D}_{\infty}^{f}(\Lambda)$. From the previous lemma it follows that there is an exact sequence of finite dimensional $\Lambda$-polydules of the form $0 \rightarrow Z \stackrel{f}{\rightarrow} M^{\prime} \oplus Z^{\prime} \stackrel{g}{\rightarrow} N \rightarrow 0$ with $m_{1}^{Z}=m_{1}^{Z^{\prime}}=m_{1}^{M^{\prime}}=0$ and where $v: Z \simeq Z^{\prime}$ and $M \simeq M^{\prime}$. We now show that this implies $M \leq \operatorname{deg} N$. 
Let $W=\operatorname{Im} f$. For each $i \in Z$, choose a $k$-complement $C^{i}$ of $W^{i}$ in $M^{i} \oplus Z^{i}$, so $W^{i} \oplus C^{i} \simeq\left(M^{\prime}\right)^{i} \oplus\left(Z^{\prime}\right)^{i}$ as $k$-vector spaces. Let $\mathcal{F}$ be the set of all strict morphisms $\tilde{f}: Z \rightarrow M^{\prime} \oplus Z^{\prime}$ such that $\tilde{f}$ is injective and $(\operatorname{Im} \tilde{f}) \cap C=0$. These are open conditions, so $\mathcal{F}$ is a variety.

For each $\tilde{f} \in \mathcal{F}$ we define an $A_{\infty}$-module structure on $C$ and a strict morphism $\tilde{g}: M^{\prime} \oplus Z^{\prime} \rightarrow C$ such that $0 \rightarrow Z \stackrel{\tilde{f}}{\rightarrow} M^{\prime} \oplus Z^{\prime} \stackrel{\tilde{g}}{\rightarrow} C \rightarrow 0$ becomes an exact sequence. Let $\tilde{f}=\left(\begin{array}{c}\tilde{\phi} \\ \tilde{\psi}\end{array}\right): Z \rightarrow W \oplus C$. We assume $\tilde{f} \in \mathcal{F}$, so $\tilde{\phi}$ is an invertible map of graded vector spaces. We define

$$
\tilde{g}=\left(\tilde{\psi}(\tilde{\phi})^{-1} \quad 1_{C}\right): W \oplus C \rightarrow C
$$

and

$$
m_{n}^{C}\left(\lambda_{n-1}, \ldots, \lambda_{1}, a\right)=\tilde{g}\left(m_{n}^{M^{\prime} \oplus Z^{\prime}}\left(\lambda_{n-1}, \ldots, \lambda_{1}, a\right)\right)
$$

for all $n \geq 2, a \in C$ and $\lambda_{1}, \ldots, \lambda_{n-1} \in \Lambda$. Since $\operatorname{dim}_{k} C^{i}=\operatorname{dim}_{k} N^{i}$ for all $i \in \mathbb{Z}$, this determines a morphism of varieties $\theta: \mathcal{F} \rightarrow \mathcal{M}_{\Lambda, d}$. This morphism is defined in such a way that $\theta(\tilde{f})$ corresponds to a polydule quasi-isomorphic to cone $\tilde{f}$.

There is an open set $U \in \mathbb{A}_{1}$ containing 0 such that the morphism $f_{(t)}=f+$ $t \cdot\left(\begin{array}{l}0 \\ v\end{array}\right): Z \rightarrow M^{\prime} \oplus Z^{\prime}$ belongs to $\mathcal{F}$ whenever $t \in U$. Except for a finite number of points in $U$, the second component of $f_{(t)}$ has full rank and cone $f_{(t)} \simeq M^{\prime} \simeq M$. If $t=0$, then cone $f_{(0)}=$ cone $f \simeq N$. So $M \leq_{\operatorname{deg}} N$.

Combining the results so far in this section we get exactly the algebraic characterisation of degeneration to which we were aiming.

Theorem 5.5. Let $M, N \in \mathcal{M}_{\Lambda, \underline{d}}$. Then the following are equivalent:

(a) $M \leq_{\operatorname{deg}} N$.

(b) There is an object $Z$ in $\mathcal{D}_{\infty}^{f}(\Lambda)$ and an exact triangle

$$
Z \rightarrow M \oplus Z \rightarrow N \rightarrow Z[1] .
$$

(c) There is an exact sequence of finite dimensional $\Lambda$-polydules of the form

$$
\begin{gathered}
0 \rightarrow Z \rightarrow M^{\prime} \oplus Z^{\prime} \rightarrow N \rightarrow 0 \\
\text { with } m_{1}^{Z}=m_{1}^{Z^{\prime}}=m_{1}^{M^{\prime}}=0 \text { and where } Z \simeq Z^{\prime} \text { and } M \simeq M^{\prime} .
\end{gathered}
$$

If $a=b=0$ and $\underline{d}=(d)$, then $\mathcal{M}_{\Lambda, \underline{d}}$ is equal to the module variety $\bmod (\Lambda, d)$. We get the Riedtmann-Zwara Theorem $([\mathrm{Z},[\underline{\mathrm{R}})$ as a corollary.

Corollary 5.6 (Riedtmann-Zwara Theorem). Let $\Lambda$ be a finite dimensional $k$ algebra and let $M$ and $N$ be two d dimensional $\Lambda$-modules. Then $M \leq{ }_{\operatorname{deg}} N$ if and only if there exists a finite dimensional $\Lambda$-module $Y$ and a short-exact sequence $0 \rightarrow Y \rightarrow M \oplus Y \rightarrow N \rightarrow 0$.

Proof. If $v: Z \rightarrow Z^{\prime}$ is an isomorphism of polydules, then it induces a bijective $\Lambda$-morphism (so a $\Lambda$-isomorphism) $v_{1}: Z^{0} \rightarrow\left(Z^{\prime}\right)^{0}$. From the equivalence between (a) and (c) in the theorem it follows that $M \leq_{\operatorname{deg}} N$ if and only if there exists a finite dimensional $\Lambda$-module $Z^{0}$ and an exact sequence of $\Lambda$-modules $0 \rightarrow Z^{0} \rightarrow$ $M \oplus Z^{0} \rightarrow N \rightarrow 0$.

In [JSZ, another theory of degeneration in derived categories was developed. There degenerations are studied in the space comproj ${ }^{d}$, a space which parameterises right bounded complexes of projective modules with so-called dimension array $\underline{d}=$ $\left(\mathrm{d}_{i}\right)$ (see [JSZ for details). There is a group G acting on this space, and G-orbits 
correspond to quasi-isomorphism classes in $\mathcal{D}(\bmod \Lambda)$ where the dimension array appears. One then defines $M \leq_{\text {top }} N$ if $N$ is in the closure of the G-orbit of $M$.

The two different approaches to making parameter spaces of objects in derived categories correspond to two alternative ways of viewing the objects. The point of view taken in [JSZ, leading to the definition of comproj ${ }^{\text {, }}$, is that the objects are complexes of projective modules. This is justified because there is a fully faithful functor from $\mathcal{D}^{b}(\bmod \Lambda)$ into $\mathcal{K}^{-}(\Lambda)$, the homotopy category of right bounded complexes of finitely generated projective $\Lambda$-modules. The alternative point of view taken in the present paper, leading to the definition of $\mathcal{M}_{\Lambda, \underline{d}}$, is to think of the objects as polydules via the equivalence $\mathcal{D}^{b}(\bmod \Lambda) \stackrel{\sim}{\longrightarrow} \mathcal{D}_{\infty}^{f}(\Lambda)$. We make a comparison between the two approaches.

The space comproj ${ }^{-\underline{d}}$ is an affine variety if and only if $\underline{d}=\left(\mathrm{d}_{i}\right)$ is bounded, meaning that $\mathrm{d}_{i}$ is non-zero only for finitely many $i$. If $\underline{\mathbf{d}}$ is not bounded, then comproj $\underline{\mathbf{d}}$ is only an inverse limit of affine varieties. Also, if $\underline{d}$ is not bounded, some of the points in comproj- correspond to complexes with unbounded homology. These difficulties can be removed if the global dimension of $\Lambda$ is finite, because in that case $\mathcal{D}^{b}(\bmod \Lambda)$ is equivalent to $\mathcal{K}^{b}(\Lambda)$, the bounded homotopy category, and there is no need to consider dimension arrays that are not bounded.

The dimension array of a complex is not preserved under quasi-isomorphism. By adding contractible complexes to any given complex $M$ in $\mathcal{K}^{-}(\Lambda)$, one obtains quasi-isomorphic complexes that have different dimension arrays, so the quasiisomorphism class of $M$ is represented in comproj ${ }^{\mathrm{d}}$ for infinitely many different d. The presence of contractible complexes might be considered a small drawback, but the value of the added flexibility they bring with them is clearly demonstrated in [JSZ, Example 4.1].

The spaces comproje do not specialise to module varieties. With some trivial exceptions, a given module variety $\bmod (\Lambda, d)$ is not equal to comproj for any dimension array $\underline{d}$. It is nevertheless possible to study degeneration of modules inside the varieties comproj .

Varieties of polydules are in many respects more similar to module varieties. The space $\mathcal{M}_{\Lambda, \underline{d}}$ is an affine variety, independent of the global dimension of $\Lambda$. Since homology dimensions can be measured by functors on the derived category, each quasi-isomorphism class belongs to a unique $\mathcal{M}_{\Lambda, \underline{d}}$. As we have seen, by setting $a=b=0$, the varieties $\mathcal{M}_{\Lambda, \underline{d}}$ do specialise to module varieties. The main new feature that appears when $a<b$ is that the group action is given by a more general form of conjugation than ordinary matrix conjugation since there are more general isomorphisms than those given by a change of basis.

An advantage of the approach using complexes is that while in $\mathcal{M}_{\Lambda, \underline{d}}$ all degenerations obviously preserve homology dimensions, in comproj ${ }^{\underline{d}}$ other kinds of degenerations are possible. On the other hand, if only degenerations that preserve homology dimensions are to be considered, then $\mathcal{M}_{\Lambda, \underline{d}}$ might be the best choice of variety. Evidence of this is the effortless proof of Lemma 4.3. while proving the corresponding result in comproj ${ }^{\mathrm{d}}$ is a harder task. The differences notwithstanding, the two concepts of degeneration are compatible in the sense of the following corollary.

Corollary 5.7. Let $X$ and $Y$ be two right bounded complexes of finitely generated projective $\Lambda$-modules with the same dimension array $\underline{\mathrm{d}}$. Suppose $\operatorname{dim}_{k} H^{i} X=$ $\operatorname{dim}_{k} H^{i} Y$ for all $i \in \mathbb{Z}$ and that $\sum_{i \in \mathbb{Z}} \operatorname{dim}_{k} H^{i} X<\infty$. Let $M_{X}$ and $M_{Y}$ be 
$\Lambda$-polydules with $m_{1}^{M_{X}}=0=m_{1}^{M_{Y}}$ which are quasi-isomorphic to $X$ and $Y$ respectively.

Then $M_{X} \leq_{\operatorname{deg}} M_{Y}$ if and only if $X \leq_{\mathrm{top}} Y$.

Proof. From the version of the Riedtmann-Zwara Theorem proved in [JSZ] it follows that the relation $X \leq_{\text {top }} Y$ can be characterised by the existence of an object $Z$ in $\left.\mathcal{D}^{b}(\bmod \Lambda)\right)$ and an exact triangle $Z \rightarrow X \oplus Z \rightarrow Y \rightarrow Z[1]$. Now the statement follows from Theorem 5.5 and the equivalence of triangulated categories $\mathcal{D}^{b}(\bmod \Lambda) \stackrel{\sim}{\longrightarrow} \mathcal{D}_{\infty}^{f}(\Lambda)$.

The proof of Theorem [5.5 is not particularly constructive. If $M$ and $N$ are two $\Lambda$-polydules and $M$ degenerates to $N$, it might not be easy to find an object $Z$ in $\mathcal{D}_{\infty}^{f}(\Lambda)$ as in part (b) of the theorem. The following example shows how to find such an object $Z$ when the degeneration is of the special type appearing in Lemma 4.3 .

Example 5.8. Let $M \in \mathcal{M}_{\Lambda, \underline{d}}$. From Lemma 4.3 we know that $M \leq_{\operatorname{deg}} H^{*} M$. We find a polydule $Z$ and a triangle $Z \rightarrow M \oplus Z \rightarrow H^{*} M \rightarrow Z[1]$ in this case. Assume that $H^{i} M$ is non-zero only in an interval $s \leq i \leq t$. We use the notation $Z_{i}=\tau={ }^{\leq i} M$ for the truncated polydules. (Truncation is straightforward when $m_{1}^{M}=0$.) We have an exact triangle

$$
\eta_{t-1}: Z_{t-1} \rightarrow M \rightarrow H^{t} M \rightarrow Z_{t-1}[1]
$$

and by induction exact triangles

$$
\eta_{i}: Z_{i} \rightarrow Z_{i+1} \rightarrow H^{i+1} M \rightarrow Z_{i}[1]
$$

for $s-1 \leq i<t$. Then by taking the direct sum $\eta=\eta_{t-1} \oplus \cdots \oplus \eta_{s-1}$ of exact triangles and letting $Z=Z_{t-1} \oplus Z_{t-2} \oplus \cdots \oplus Z_{s} \oplus Z_{s-1}$, where $Z_{s-1}=0$, we have the exact triangle

$$
\eta: Z \rightarrow M \oplus Z \rightarrow H^{*} M \rightarrow Z[1]
$$

We now give the proof of Lemma 4.4 .

Proof of Lemma 4.4. Assume $N \in \overline{\mathcal{G}_{Q} \cdot M}$. We have $\mathcal{G}_{Q} \cdot M \subseteq \mathcal{G} \cdot M$ and therefore $\overline{\mathcal{G}_{Q} \cdot M} \subseteq \overline{\mathcal{G} \cdot M}$. Hence $M \leq_{\operatorname{deg}} N$.

Now assume that $M \leq_{\operatorname{deg}} N$. So there exists a polydule $Z$ in $\mathcal{D}_{\infty}^{f}(\Lambda)$ and an exact triangle

$$
Z \rightarrow M \oplus Z \rightarrow N \rightarrow Z[1]
$$

by Theorem 5.2 We may assume that $Z$ is a polyrepresentation. Now, by the proof of Theorem $\left[5.4\right.$ there exists a family of representations $M_{t}$ in $\overline{\mathcal{G}_{Q} \cdot M}$, such that $M_{t} \cong M$ for all except a finite number of $t$, and $M_{0} \cong N$. This shows that $N \in \overline{\mathcal{G}_{Q} \cdot M}$.

\section{REFERENCES}

[ARS] M. Auslander, I. Reiten, S. O. Smalø, Representation theory of Artin algebras. Cambridge Studies in Advanced Mathematics, 36. Cambridge University Press, Cambridge, 1997. xiv+425 pp. MR $1476671(98 \mathrm{e}: 16011)$

[GO'H] F. Grünewald, J. O'Halloran, A characterization of orbit closure and applications, J. Algebra 116 (1988), no. 1, 163-175. MR944153(89j:17006)

$[\mathrm{H}]$ D. Happel, Triangulated categories in the representation theory of finite-dimensional algebras. London Mathematical Society Lecture Note Series, 119. Cambridge University Press, Cambridge, 1988. x+208 pp. MR935124 (89e:16035) 
[JK] G. Janelidze, G. M. Kelly, A note on actions of a monoidal category, Theory Appl. Categ. 9 (2001/02), 61-91 (electronic). MR.1897810 (2003f:18007)

[JSZ] B. T. Jensen, X. Su, A. Zimmermann, Degenerations for derived categories, J. Pure Appl. Algebra 198 (2005), no. 1-3, 281-295. MR2133687 (2006a:18009)

[K] B. Keller, Introduction to A-infinity algebras and modules, Homology Homotopy Appl. 3 (2001), no. 1, 1-35. MR1854636 (2004a:18008a)

[L-H] K. Lefèvre-Hasegawa, Sur les $A_{\infty}$-catégories, Thèse de Doctorat, Université Paris 7, 2003, arXiv:math.CT/0310337.

[R] C. Riedtmann, Degenerations for representations of quivers with relations, Ann. Sci. École Norm. Sup. (4) 19 (1986), no. 2, 275-301. MR868301 (88b:16051)

[W] C. A. Weibel, An introduction to homological algebra. Cambridge Studies in Advanced Mathematics, 38. Cambridge University Press, Cambridge, 1994. xiv+450 pp. MR.1269324 (95f:18001)

[Y] Y. Yoshino, On degeneration of modules, J. Algebra 278 (2004), no. 1, 217-226. MR:2068075 (2005d:16026)

[Z] G. Zwara, Degenerations of finite dimensional modules are given by extensions, Compositio Math. 121 (2000), no. 2, 205-218. MR1757882 (2001g:14077)

Institutt for matematiske fag, Norwegian University of Science and Technology, NO-7491 Trondheim, NORWAY

E-mail address: berntj@math.ntnu.no

Current address: Institut de Mathematique de Jussieu, UMR 7586, 175 Rue Chevaleret, 75013 Paris, France

Institutt for matematiske fag, Norwegian University of Science and Technology, NO-7491 TRONDHEIM, NORWAY

E-mail address: dagma@math.ntnu.no

Current address: Department of Mathematics, Syracuse University, 215 Carnegie Hall, Syracuse, New York 13244

Mathematisches Institut, Universität Zu Köln, Weyertal 86-90, 50931 Köln, Germany

E-mail address: xsu@math.uni-koeln.de

Current address: Mathematical Sciences, University of Bath, Claverton Down, Bath, BA2 7AY, United Kingdom 